Open Access

\title{
Thrombocytopenia-absent radius (TAR) syndrome due to compound inheritance for a 1q21.1 microdeletion and a low- frequency noncoding RBM8A SNP: a new familial case
}

\author{
Elisa Tassano ${ }^{1 *}$, Stefania Gimelli ${ }^{2}$, Maria Teresa Divizia ${ }^{3}$, Margherita Lerone ${ }^{3}$, Carlotta Vaccari ${ }^{4}$, Aldamaria Puliti ${ }^{3,4}$ \\ and Giorgio Gimelli ${ }^{1}$
}

\begin{abstract}
Background: Thrombocytopenia-absent radius syndrome (TAR; MIM 274000) is a rare autosomal recessive disorder combining specific skeletal abnormalities with a reduced platelet count. TAR syndrome has been associated with the compound inheritance of an interstitial microdeletion in 1q21.1 and a low frequency noncoding RBM8A SNP.

Results: Here, we report on a patient with scapulo-humeral hypoplasia, bilateral radio-ulnar agenesis with intact thumbs, bilateral proximal positioning of the first metacarpal, bilateral fifth finger clinodactyly, bilateral radial deviation of the hands, and thrombocytopenia. Molecular studies showed compound heterozygosity for the 1q21.1 microdeletion and the RBM8A rs139428292 variant in hemizygous state, inherited from the father and the mother, respectively. A second aborted fetus presented TAR features and 1q21.1 microdeletion.

Discussion: The complex inheritance pattern resulted in reduced expression of $\mathrm{Y} 14$, the protein encoded by $R B M 8 A$, and a component of the core exon-junction complex (EJC) in platelets. Further studies are needed to explain how Y14 insufficiency and subsequent defects of the EJC could cause the skeletal, haematological and additional features of TAR syndrome. In this study, we discuss other factors that could influence the overall phenotype of patients affected by TAR syndrome.
\end{abstract}

Conclusion: In this study, we discuss other factors that could influence the overall phenotype of patients affected by TAR syndrome.

Keywords: 1q21.1 microdeletion, TAR syndrome, Array-CGH, RBM8A SNPS

\section{Background}

Since the introduction of array-CGH analysis, the reported frequency of genomic imbalances associated with specific phenotypes has dramatically increased. Copy number variations $(\mathrm{CNV})$ with incomplete penetrance and variable expressivity have been described in various disorders [1-5]. The association between genomic imbalances and pathological traits can be variable, and additional genetic variations could contribute to the phenotype.

\footnotetext{
* Correspondence: eli.tassano@gmail.com

'Laboratorio di Citogentica, Istituto Giannina Gaslini, L.go G.Gaslini 5, 16147

Genoa, Italy

Full list of author information is available at the end of the article
}

In addition, several other possibilities as epigenetic phenomena, expression or regulatory variation among genes in the vicinity of the unbalanced region, the unmasking of recessive alleles and the possibility of a "two-hit" model, as proposed by Girirajan et al. [6], may account for the phenotypic variability of some genomic diseases.

Human chromosome 1 is rich in segmental duplications, particularly within the pericentromeric region [7-10]. This fact may result in the susceptibility of this region to both pathological and non-pathological CNVs that might have an evolutionary significance.

TAR syndrome (Thrombocytopenia -Absent-Radius) syndrome (MIM 274000) is characterized by thrombocytopenia 
that may be episodic, congenital skeletal deformities including bilateral absence of radius, shortening and deformity of the ulnae, and occasionally absence of all the long bones in the arm. The fingers and thumbs are always present, while other skeletal anomalies are frequent [11].

A chromosome 1q21.1 microdeletion was identified in 30 patients affected by TAR syndrome [12]. This microdeletion is mediated by Low Copy Repeats (LCRs) that can be at the basis of recurrent DNA rearrangements such as deletions, duplications and inversions through chromosome or chromatid misalignment followed by non-allelic homologous recombination (NAHR) [13-15].

TAR syndrome has a complex pattern of inheritance associated with a minimal common interstitial microdeletion of $200 \mathrm{~Kb}$ on chromosome 1q21.1. In several cases, it is inherited from an unaffected parent, while in others it is originated de novo and the presence of a 1q21.1 microdeletion is necessary but not sufficient to cause the phenotype.

Recently, it has been shown that compound inheritance of a rare null allele and one of the two low-frequency noncoding SNPs (rs139428292 or rs201779890) in RBM8A are crucial for TAR syndrome [16].

Here, we describe the clinical, cytogenetic and molecular features of a 4-month-old boy with TAR syndrome due to co-segregation of 1q21.1 microdeletion and rs139428292. An aborted fetus in the same family presented the same phenotypic features and 1q21.1 microdeletion. We discuss here whether other factors could influence the overall phenotype of TAR syndrome.

\section{Case report}

The family tree is depicted in Fig. 1.

\section{Patient 1}

The child (II-2) (Fig. 2a) is the first male child of apparently healthy nonconsanguineous parents. The mother and the father were 33 and 45 years old respectively at the time of his birth. Fetal movements were poor. Routine ultrasound examination was normal until 23 weeks of gestation when bilateral radial agenesis was demonstrated. The child was born post-term at 43 weeks of gestation by normal vaginal delivery. Birth weight was $2810 \mathrm{~g}$ (10th-25th centile). He was admitted to our institute at 4 months of age. Physical examination showed good nutritional status, forehead and right cheek telangiectasia, scapulo-humeral hypoplasia, bilateral radio-ulnar agenesis with intact thumbs, bilateral proximal positioning of the first metacarpal, bilateral fifth finger clinodactyly and bilateral radial deviation of the hands. X-ray confirmed all these skeletal findings. The child also presented thrombocytopenia (40.000/ mmc), PTT (43.7 s). The phenotypic features were characteristic of TAR syndrome (MIM 274000).

\section{Patient 2 (Fetus)}

During the third pregnancy (II-3) (Fig. 1b), ultrasound examination was performed at 11 weeks of gestation, suggesting the presence of upper limb anomalies. This finding was confirmed at 15 th week of gestation. The couple opted for termination of pregnancy. The chromosomal analysis of amniotic cells (400 bands) excluded any visible abnormality. Post-mortem examination of the fetus demonstrated bilateral radial agenesis (Fig. 2b). The mother had a previous pregnancy, which ended in spontaneous abortion at the $2^{\text {nd }}$ month of gestation (II-1). There was no exposure to alcohol, smoking or

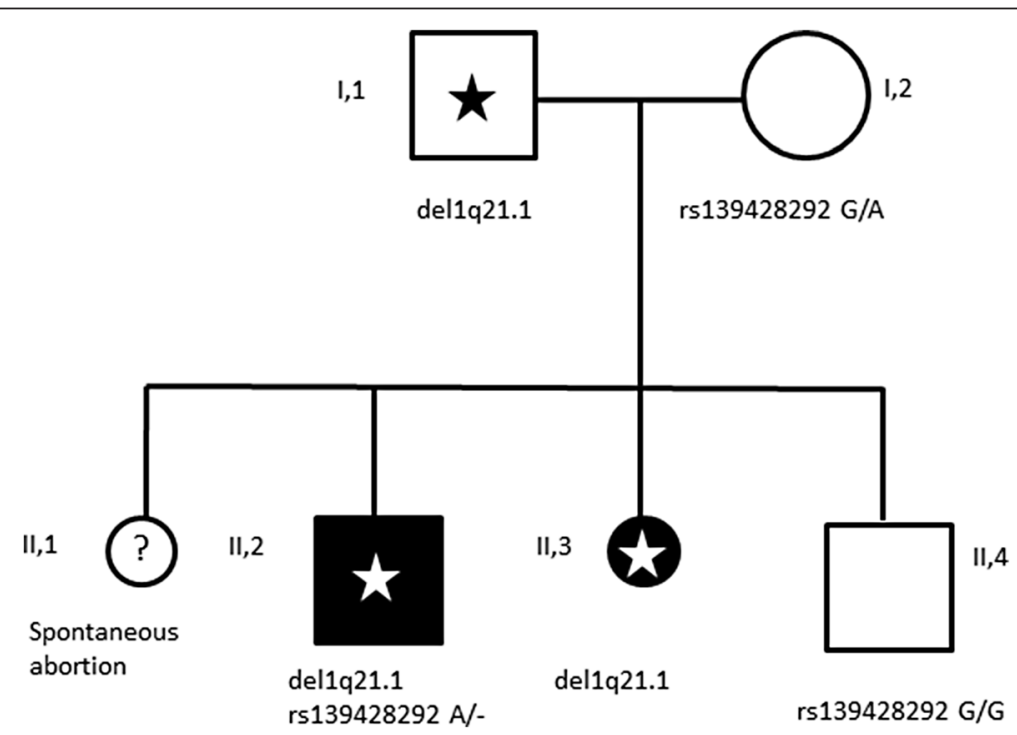

Fig. 1 Genealogical tree of the family with TAR syndrome 

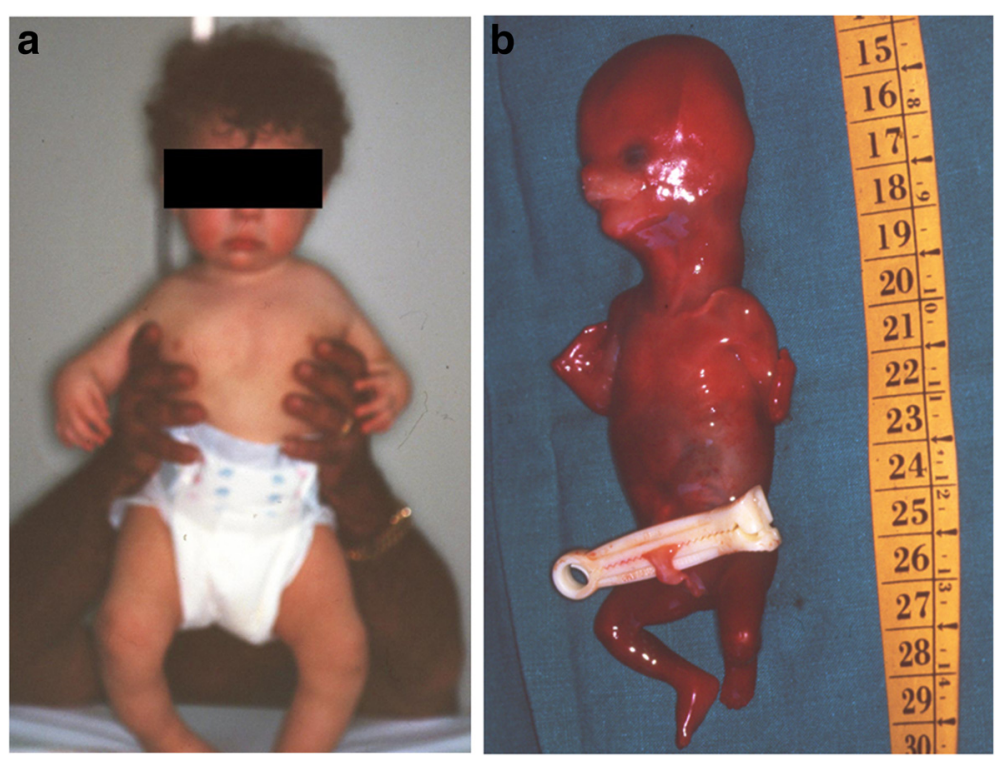

Fig. 2 a The proband (II-2); bilateral absence of radius with thumb conservation and bilateral genu varum. b The fetus (II-3); bilateral radial aplasia

infections during pregnancy. A subsequent pregnancy resulted in the delivery of a healthy child (II-4).

\section{Results}

All patients and their parents showed a normal karyotype. Array-CGH analysis was performed on the available members of the family. An identical 1q21.1 microdeletion $(\sim 539 \mathrm{~Kb})$ [arr 1q21.1(145,291,711-145,831,389)x1] was identified in the child with phenotypic features of TAR syndrome (II, 2) as showed in Fig. 3a. The microdeletion was confirmed by FISH (Fig. 3b). The same microdeletion was also present in his apparently normal father (I, 1). An elective abortion was performed because of ultrasound findings of upper limb anomalies, strongly suggestive of TAR syndrome (II, 3). FISH analysis by BAC RP11105E14 (chr1:145,474,158-145,636,051) on archived specimens confirmed the presence of the "TAR microdeletion" (Fig. 3c). In the same family, one spontaneous abortion (II, 1) was reported but unfortunately not investigated, as biological specimens were not available. The deleted region contains 12 MIM genes: NBPF20 (MIM 614007; neuroblastoma breakpoint family, member 20), NBPF10 (MIM 614000; neuroblastoma breakpoint family, member 10), NBPF9 (MIM 613999; neuroblastoma breakpoint family, member 9), HFE2 (MIM 602390; hemochromatosis type 2A), TXNIP (MIM 606599; thioredoxin-interacting protein), RBM8A (MIM 605313; RNA-binding motif protein 8A), GNRHR2 (MIM 612875; gonadotropin-releasing hormone receptor 2), PEX11B (MIM 603867; peroxisome biogenesis factor 11B), ITGA10 (MIM 604042; integrin, alpha-10), PIAS3 (MIM 605987; protein inhibitor of activated STAT3), CD160 (MIM 604463; CD160 antigen), PDZK1 (MIM 603831; PDZ domain-containing 1). It is interesting to note the presence of the LIX1L (Gene ID: 128077; Lix1 homolog (chicken) like) gene (Fig. 3d).

The family were analysed for the rs139428292 (G > A) and the rs201779890 (G > C) SNPs of RBM8A gene by direct sequencing. The analysis of rs139428292 showed that the patient harboured the minor (A) allele, which was inherited from his healthy mother. The father and the healthy brother were both homozygous for the major (G) allele. All family members carried the major (G) allele of rs201779890 in a homozygous state (Fig. 4).

\section{Discussion}

We describe an identical 1q21.1 microdeletion in affected and non-affected members of a family with TAR syndrome. The same rearrangement was firstly described in 30 patients affected by Thrombocytopenia-AbsentRadius (TAR) syndrome (MIM 274000), a rare malformation syndrome characterized by hypo-megakaryocytic thrombocytopenia and bilateral absence of the radius in the presence of both thumbs [12]. The microdeletion was inherited from either the unaffected mother or the unaffected father in $75 \%$ of cases. Recently, it has been proposed to consider this syndrome as a complex trait disease requiring at least two genetic changes: the rare microdeletion and another, relatively frequent, genetic variation that acts as a modifier of the other one. The family described in this study was referred to our centre for TAR syndrome and was investigated by array-CGH. A 1q21.1 microdeletion of $\sim 539 \mathrm{~Kb}$ overlapping the TAR critical region, inherited from the phenotypically normal father, was identified in the two affected cases (II, 2 and II, 3). The identification of the interstitial microdeletion 


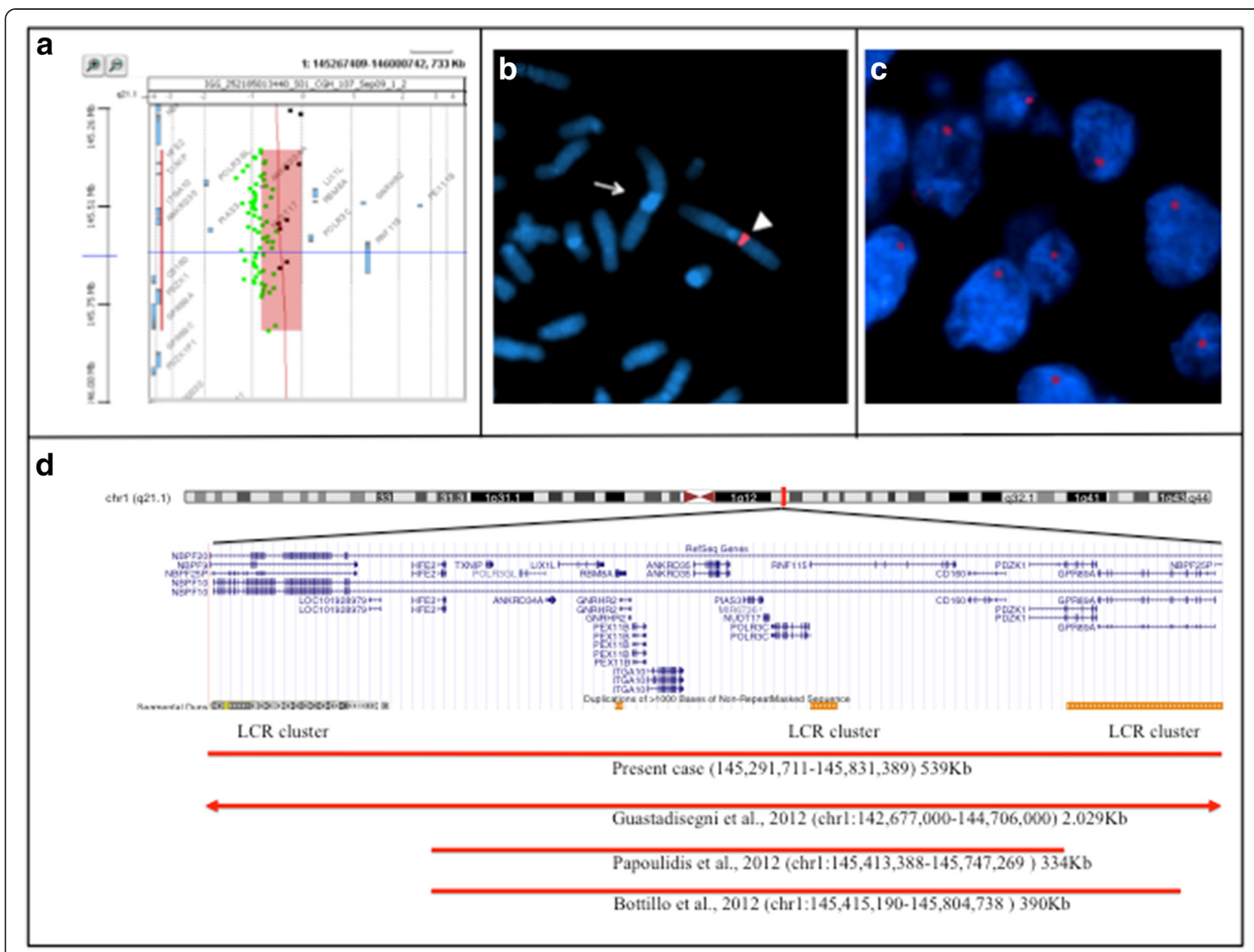

Fig. 3 Results of array-CGH and FISH analyses. a Array-CGH analysis shows a $\sim 539 \mathrm{~Kb}$ microdeletion at 1q21.1 band inherited from the father. b FISH confirmation of a hemizygous interstitial 1q21.1 deletion using a BAC probe RP11-105E14 (chr1:145,474,158-145,636,051) (red). c Interphase FISH with RP11-105E14 (chr1:145,474,158-145,636,051) (red) on deparaffinized fetal tissue from patient II, 3. Nuclei show a unique red signal indicating the presence of the deletion. $\mathbf{d}$ Overview of the 1q21.1 region and its genes and LCRs contents, according to the UCSC Genome Browser (GRCh37/ hg19 assembly). The bars indicate the deleted region (red) in our patient and the deleted regions in patients reported by Guastadisegni et al. [28], Papoulidis et al. [30] and Bottillo et al. [29].

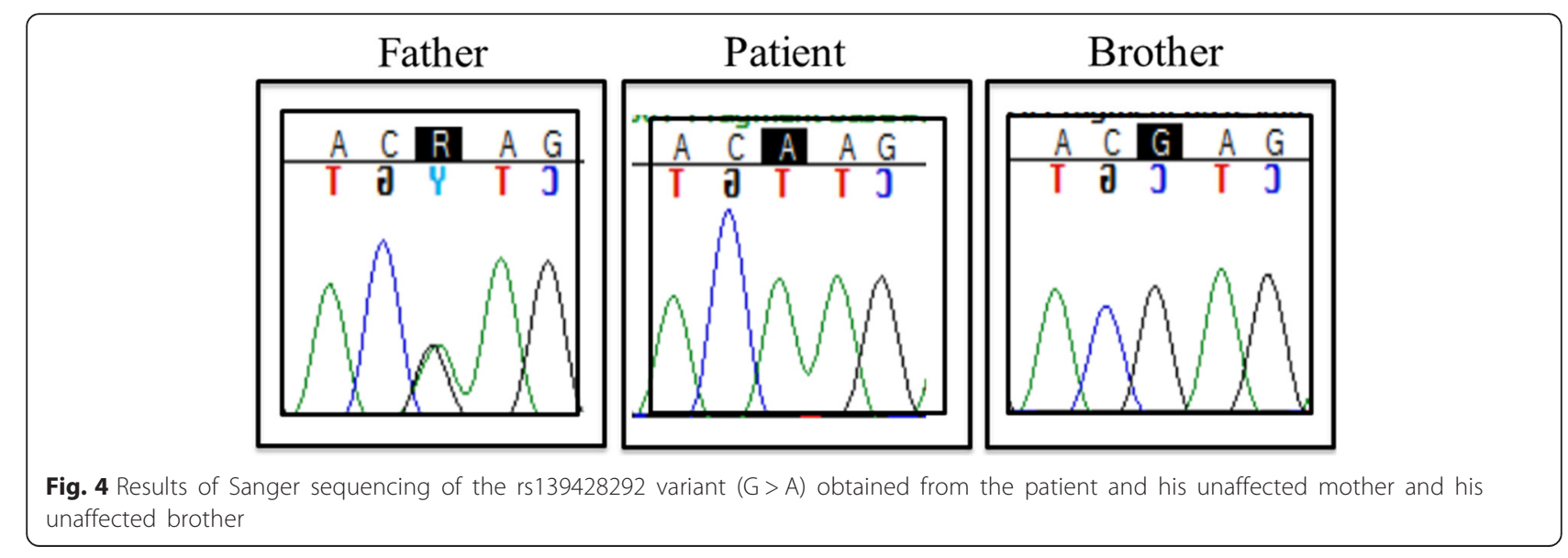


in affected offspring and the paternal inheritance are in agreement with the findings of Klopocki and collaborators [12]. The presence of $1 \mathrm{q} 21.1$ microdeletion is necessary but not sufficient to cause the phenotype, since, in the majority of cases, TAR traits can develop only in the presence of a second modifier as the low-frequency regulatory SNP reported by Albers et al. [16].

In fact, a study identified two rare single nucleotide polymorphisms (SNPs) in the regulatory region of the RBM8A gene that are involved in TAR syndrome through the reduction of the expression of the RBM8Aencoded Y14 protein [16]. The first allele (rs139428292 $\mathrm{G}>\mathrm{A}$ ), which is located in the $5^{\prime}$ untranslated region (UTR) of the gene, was demonstrated to have a minor allele frequency (MAF) of $3.05 \%$, and the second allele (rs201779890 G > C), located in the first intron of the gene, exhibited a MAF of $0.42 \%$, in 7504 healthy individuals from Cambridge BioResource (Cambridge, UK) $[16,17]$. Our patient had inherited a low-frequency 5' UTR SNP (rs139428292 G > A) from his mother and the 1q21.1 microdeletion from his father.

A number of patients with TAR syndrome were reported, but in almost all these cases the precise coordinates of the deleted regions were not available and in others no mutation analysis of RBM8A SNP had been performed [18-27].

At our knowledge, in only three TAR cases with 1q21.1 deletion, analysed by array-CGH, the precise coordinates of the deletion has been reported [28-30] (Fig. 3d). Two are prenatal cases and one postnatal. All have the classical TAR features (thrombocytopenia, upper limbs and hands anomalies); two have inherited the low-frequency 5' UTR SNP (rs139428292 G>A) and the minimal overlapping 1q21.1 deletion region ranging from $145,415,190$ to $145,747,269$ [29, 30].

Particularly, the child affected by TAR syndrome associated with Langerhans cell histiocytosis described by Guastadisegni et al. [28] showed a larger deletion (2.029 Kb) and a significant downregulation of the commonly deleted genes. The mainly implicated gene in the syndrome is $R B M 8 A$, a gene encoding the exon-junction complex subunit member Y14. Y14 is a small protein with an RNAbinding domain and one of the four components of EJC, which is involved in basic cellular functions such as nuclear export and subcellular localization of specific transcripts, translational enhancement, nonsense-mediated RNA decay and splicing [31]. It has a crucial role during embryonic developmental [32]. The level of Y14 was found to be significantly lower in the platelets of TAR patients. It is noteworthy, that Y14 may regulate the expression of genes involved in the proliferation of hematopoietic cells. However, it is not clear how a deficiency in Y14 exerts its effects at a cellular level and in particular how it affects the production of megakaryocytes and platelets. A possible explanation for this observation could be that deficiency in EJC could have an influence on the defective cell signalling in megakaryocyte. Similarly, no relation has been found between RBM8A deletion and TAR skeletal anomalies. To provide an explanation for the skeletal abnormalities observed in TAR syndrome, Albers et al., [24] assumed that, in addition to a tissue-dependent effect, it is possible that the regulatory SNPs had developmental stage-dependent consequences keeping as an example the Mecom gene encoding Evil that is expressed in a transient manner in emerging limb buds in mouse [33].

Albers et al., [24] explained that TAR phenotype could be also influenced from other factors such as environmental factors altering gene expression, incomplete penetrance or additional modifier alleles. We speculate that other genes in the 1q21.1 region other than $R B M 8 A$ could influence the phenotype of TAR syndrome. It is interesting to note that, among the several genes with a known function located within the region, the PIAS3 gene could be indicated as the most conspicuous candidate for thrombocytopenia and the Lix1L gene, known be transiently expressed during chick hind-limb development, could be proposed as the candidate gene for limb abnormalities [34-36].

\section{Conclusions}

In conclusion, we reported on a new familial case of TAR syndrome in a child and in a fetus carrying a 1q21.1 microdeletion and the low-frequency 5'UTR SNP (rs139428292 G>A). We also focused on two genes (PIAS3 and Lix1L), contained in the deleted region, which could play a role in determining some important aspects of the phenotype of this syndrome. Obviously, further in-depth studies are needed to clarify the possible role of these genes.

\section{Methods \\ Cytogenetics, fluorescent in situ hybridization and array- CGH analyses}

Karyotypes were performed on peripheral blood of patients and their parents. Fluorescent in situ hybridization (FISH) analysis was performed following the manufacturer's instructions (Vysis, Abbott Molecular, Illinois, U.S.A.). BAC clone was selected from the human library RPCI-11 according to the UCSC Human Genome Assembly (GRCh37/hg19). Array-CGH using the Human CGH Kit 244 K (Agilent Technologies, Palo Alto, CA, U.S.A.) covering the whole genome with a $8.9 \mathrm{~Kb}$ overall median probe spacing was performed following the manufacturer's protocol.

\section{FISH analysis on paraffin-embedded fetal tissues}

To prepare paraffin-embedded tissue sections fixed on positively charged slides we cut $4-5 \mathrm{~m} \mu$ thick paraffin 
sections using a microtome. Floating sections were mounted on positively charged slides. Slides were treated by Paraffin Pretreatment Kit (Abbott Molecular Inc., IL, USA) to deparaffinise specimens. Then, the slides were treated with protease and hybridization with BAC probes was performed according to the appropriate Vysis protocol.

\section{Genotyping of 5' UTR and intronic SNP of the RBM8A gene}

The genotypes of the rs139428292 and the rs201779890 SNPs were obtained. Genomic DNA was isolated from peripheral blood leukocytes using a standard protocol. The genomic regions encompassing the two SNPs were PCR amplified, purified and then sequenced on both strands using the BigDye dideoxy-terminator chemistry on an ABI 3100 DNA sequencer (Applied Biosystems, Foster City, CA). Primers used for both amplification and sequencing were the following: rs139428292 (Fw: CCTTTCCCCTCTGCGACA; Rv: CCCAGCCTCGTGA AGATCTA) and rs201779890 (Fw: TAGATCTTCACGAGGCTGGG; Rv: GGGGCGGAATCTCTAATCCA).

\section{Ethics Statement}

The current study was performed using peripheral blood of the members of the family treated at the Istituto Giannina Gaslini, Genova, Italy. The parents of the patient gave written informed consent allowing molecular and genetic studies. We didn't request approval by Review Board of our institution, because our study request only classical and molecular cytogenetic analyses. For cytogenetics analyses are sufficient only written informed consent of the parents (DM 21 dicembre 2007). The informed consents of the parents were previously authorized by the Review Board of our institution. We didn't conduct research outside our country of residence. We didn't approach the local authorities before beginning work on this study. The full name of the ethics committee of our institution is Comitato di Etica per la Ricerca Scientifica Biomedica, per la Buona Pratica Clinica e per la Sperimentazione dei Farmaci.

\section{Consent}

Written informed consent was obtained from the patient's parents for the publication of this report and any accompanying images.

\section{Competing interests}

The authors declare that they have no competing interests.

\section{Authors' contributions}

All authors have made substantial contributions to conception and design, acquisition of data, analysis and interpretation of data. All authors have been involved in drafting the manuscript and revising it critically for important intellectual content. All authors read and approved the final manuscript.

\section{Acknowledgments}

We thank the patient's parents for their kind participation and support. We thanks Marco Bertorello and Corrado Torello for technical supports. This work was supported by "Cinque per mille dell'IRPEF- Finanziamento della ricerca sanitaria" and "Finanziamento Ricerca Corrente, Ministero Salute (contributo per la ricerca intramurale).

\section{Author details}

'Laboratorio di Citogentica, Istituto Giannina Gaslini, L.go G.Gaslini 5, 16147 Genoa, Italy. ${ }^{2}$ Service of Genetic Medicine, University Hospitals of Geneva, 1211 Geneva, Switzerland. ${ }^{3}$ U.O.C. Medical Genetics, Istituto Giannina Gaslini, 16147 Genoa, Italy. ${ }^{4}$ DiNOGMI, University of Genoa, 16147 Genoa, Italy.

Received: 27 July 2015 Accepted: 27 October 2015

Published online: 05 November 2015

\section{References}

1. Kirov G. A The role of copy number variation in schizophrenia. Expert Rev Neurother. 2010;10:25-32.

2. Hannes FD, Sharp AJ, Mefford HC, de Ravel T, Ruivenkamp CA, Breuning $\mathrm{MH}$, et al. Recurrent reciprocal deletions and duplications of 16p13.11: the deletion is a risk factor for MR/MCA while the duplication may be a rare benign variant. J Med Genet. 2009;46:223-32.

3. Mefford HC, Muhle H, Ostertag P, von Spiczak S, Buysse K, Baker C, et al. Genome-wide copy number variation in epilepsy: novel susceptibility loci in idiopathic generalized and focal epilepsies. PLoS Genet. 2010;6:e1000962.

4. Vassos E, Collier DA, Holden S, Patch C, Rujescu D, St Clair D, et al. Penetrance for copy number variants associated with schizophrenia. Hum Mol Genet. 2010;19:3477-81.

5. Walters RG, Jacquemont $S$, Valsesia A, de Smith AJ, Martinet D, Andersson J, et al. A new highly penetrant form of obesity due to deletions on chromosome 16p11.2. Nature. 2010;463:671-5.

6. Girirajan S, Rosenfeld JA, Cooper GM, Antonacci F, Siswara P, Itsara A, et al. A recurrent 16p12.1 microdeletion supports a two-hit model for severe developmental delay. Nat Genet. 2010;42:203-9.

7. Bailey JA, Yavor AM, Massa HF, Trask BJ, Eichler EE. Segmental duplications: organization and impact within the current human genome project assembly. Genome Res. 2001;11:1005-17.

8. Bailey JA, Gu Z, Clark RA, Reinert K, Samonte RV, Schwartz S, et al. Recent segmental duplications in the human genome. Science. 2002;297:1003-7.

9. Cheung J, Estivill X, Khaja R, MacDonald JR, Lau K, Tsui LC, et al. Genomewide detection of segmental duplications and potential assembly errors in the human genome sequence. Genome Biol. 2003;4:R25.

10. She X, Jiang Z, Clark RA, Liu G, Cheng Z, Tuzun E, et al. Shotgun sequence assembly and recent segmental duplications within the human genome. Nature. 2004:431:927-30.

11. Hall J, Levin J, Kuhn J, Ottenheimer E. Berkum Kv, McKusick V. Thrombocytopenia with absent radius (TAR). Medicine. 1969;48:411-39.

12. Klopocki E, Schulze H, Strauss G, et al. Complex inheritance pattern resembling autosomal recessive inheritance involving a microdeletion in thrombocytopenia-absent radius syndrome. Am J Hum Genet. 2007;80:232-40.

13. Lupski JR. Genomic disorders: structural features of the genome can lead to DNA rearrangements and human disease traits. Trends Genet. 1998;14:417-22.

14. Stankiewicz P, Lupski JR. Genome architecture, rearrangements and genomic disorders. Trends Genet. 2002;18:74-82.

15. Lupski JR, Stankiewicz P. Genomic disorders: the genomic basis of disease. Totowa: Humana Press; 2006.

16. Albers CA, Paul DS, Schulze H, Freson K, Stephens JC, Smethurst PA, et al. Compound inheritance of a low-frequency regulatory SNP and a rare null mutation in exon-junction complex subunit RBM8A causes TAR syndrome. Nat Genet. 2012:44:435-9.

17. Soranzo N, Spector TD, Mangino M, et al. A genome-wide meta-analysis identifies 22 loci associated with eight hematological parameters in the HaemGen consortium. Nat Genet. 2009;41:1182-90.

18. Bonsi L, Marchionni C, Alviano F, Lanzoni G, Franchina M, et al. Thrombocytopenia with absent radii (TAR) syndrome: from hemopoietic progenitor to mesenchymal stromal cell disease? Exp Hematol. 2009;37:1-7.

19. Giordano P, Cecinati V, Grassi M, Giordani L, De Mattia D, Santoro N. Langerhans cell histiocytosis in a pediatric patient with thrombocytopeniaabsent radius syndrome and 1q21.1 deletion: case report and proposal of a 
rapid molecular diagnosis of 1q21.1 deletion. Immunopharmacol Immunotoxicol. 2011;33:754-8.

20. Houeijeh A, Andrieux J, Saugier-Veber P, David A, Goldenberg A, Bonneau $D$, et al. Thrombocytopenia-absent radius (TAR) syndrome: a clinical genetic series of 14 further cases. Impact of the associated 1q21.1 deletion on the genetic counselling. Eur J Med Genet. 2011;54:e471-7.

21. Toriello HV. Thrombocytopenia-absent radius syndrome. Semin Thromb Hemost. 2011:37:707-12

22. Rosenfeld JA, Traylor RN, Schaefer GB, McPherson EW, Ballif BC, Klopocki E, et al. 1q21.1 Study Group. Proximal microdeletions and microduplications of 1q21.1 contribute to variable abnormal phenotypes. Eur J Hum Genet. 2012;20:754-61.

23. Albers CA, Paul DS, Schulze H, Freson K, Stephens JC, Smethurst PA. Compound inheritance of a low-frequency regulatory SNP and a rare null mutation in exon-junction complex subunit RBM8A causes TAR syndrome. Nat Genet. 2012;44:435-9. S1-2.

24. Albers CA, Newbury-Ecob R, Ouwehand WH, Ghevaert C. New insights into the genetic basis of TAR (thrombocytopenia-absent radii) syndrome. Curr Opin Genet Dev. 2013;23:316-23.

25. Baken L, Groenenberg IA, Hoogeboom AJ, Koning AH, Exalto N. Firsttrimester diagnosis of thrombocytopenia-absent radius syndrome using virtual reality. Clin Dysmorphol. 2014;23:71-3.

26. Yassaee VR, Hashemi-Gorji F, Soltani Z, Poorhosseini SM. A new approach for molecular diagnosis of TAR syndrome. Clin Biochem. 2014;47:835-9.

27. Kumar C, Sharma D, Pandita A, Bhalerao S. Thrombocytopenia absent radius syndrome with Tetralogy of Fallot: a rare association. Int Med Case Rep J. 2015;8:81-5.

28. Guastadisegni MC, Roberto R, L'Abbate A, Palumbo O, Carella M, Giordani L, et al. Thrombocytopenia-absent-radius syndrome in a child showing a larger 1q21.1 deletion than the one in his healthy mother, and a significant downregulation of the commonly deleted genes. Eur J Med Genet. 2012:55:120-3.

29. Bottillo I, Castori M, De Bernardo C, Fabbri R, Grammatico B, Preziosi N, et al. Prenatal diagnosis and post-mortem examination in a fetus with thrombocytopenia-absent radius (TAR) syndrome due to compound heterozygosity for a 1q21.1 microdeletion and a RBM8A hypomorphic allele: a case report. BMC Res Notes. 2013;22:376.

30. Papoulidis I, Oikonomidou E, Orru S, Siomou E, Kontodiou M, Eleftheriades $\mathrm{M}$, et al. Prenatal detection of TAR syndrome in a fetus with compound inheritance of an RBM8A SNP and a $334 \mathrm{~Kb}$ deletion: a case report. Mol Med Rep. 2014;9:163-5.

31. Chuang TW, Lee KM, Tarn WY. Function and Pathological Implications of Exon Junction Complex Factor Y14. Biomol. 2015;5:343-55.

32. Haremaki T, Sridharan J, Dvora S, Weinstein DC. Regulation of vertebrate embryogenesis by the exon junction complex core component Eif4a3. Dev Dyn. 2010;239:1977-87.

33. Perkins AS, Mercer JA, Jenkins NA, Copeland NG. Patterns of Evi-1 expression in embryonic and adult tissues suggest that Evi-1 plays an important regulatory role in mouse development. Development. 1991:111:479-87.

34. Rodel B, Tavassoli K, Karsunky H, Schmidt T, Bachmann M, Schaper F, et al. The zinc finger protein Gfi-1 can enhance STAT3 signaling by interacting with the STAT3 inhibitor PIAS3. EMBO J. 2000;19:5845-55.

35. Hsiao HH, Liu YC, Yang MY, et al. Decreased expression of PIAS1 and PIAS3 in essential thrombocythemia patients. Genet Mol Res. 2013;12:5617-22.

36. Swindell EC, Moeller C, Thaller C, Eichele G. Cloning and expression analysis of chicken Lix1, a founding member of a novel gene family. Mech Dev. 2001;109:405-8.

\section{Submit your next manuscript to BioMed Central and take full advantage of:}

- Convenient online submission

- Thorough peer review

- No space constraints or color figure charges

- Immediate publication on acceptance

- Inclusion in PubMed, CAS, Scopus and Google Scholar

- Research which is freely available for redistribution 